\title{
Las mujeres y la economía social y solidaria: construyendo una nueva economía
}

\section{Women and the social and solidarity economy: building a new economy}

DOI: $10.46932 / \mathrm{sfjdv} 2 \mathrm{n} 3-040$

Received in: May 1st, 2021

Accepted in: Jun 30th, 2021

\author{
Rangel Gómez, Sheila Janet \\ Maestra en Gobierno y Gestión Local \\ Universidad Estatal de Bolívar \\ Av. Ernesto Che Guevara s/n y Av. Gabriel Secaira, Guaranda, Ecuador. \\ E-mail: srangel@ueb.edu.ec \\ Rea García, Fernando Fredi \\ Maestro en Gobierno y Gestión Local \\ Universidad Estatal de Bolívar \\ Av. Ernesto Che Guevara s/n y Av. Gabriel Secaira, Guaranda, Ecuador. \\ E-mail: frea@ueb.edu.ec \\ Navas-Montes, Yonaiker del Mar \\ Doctora en Ciencias Pedagógicas \\ Universidad Estatal de Bolívar \\ Av. Ernesto Che Guevara s/n y Av. Gabriel Secaira, Guaranda, Ecuador. \\ E-mail: ynavas@ueb.edu.ec
}

\section{RESUMEN}

A lo largo de la historia, sobre todo en el ámbito local, el papel que llevaron a cabo las mujeres fue el de ser amas de casa, madres y esposas, además de proveer de alimentos. Sin embargo, en los últimos años se ha discutido y estudiado sobre si estas actividades son parte de lo privado y reproductivo o realmente pertenecen a lo público y productivo. Es así como, desde la perspectiva de género y de la economía feminista se pueden analizar estas proposiciones y visualizar los papeles que se designan a los hombres y a las mujeres en una sociedad, para de esta manera determinar las formas de comportamiento en lo social, jurídico, económico, cultural o en la vida cotidiana y rastrear el origen de tales condiciones.

La presente investigación utiliza la metodología del análisis bibliográfico, mediante el cual se recopila el concepto y teória de la Economía Social y Solidaria y su conexión con la Economía Feminista, que, en conclusión, logran complementar y apuestan por una reorganización del trabajo en el que se valorice el trabajo de las mujeres en particular reconociendo la construcción de una nueva economía.

Palabras clave: Economía Feminista, Economía, Género, Mujeres, Trabajo.

\begin{abstract}
Throughout history, especially at the local level, the role of women was to be housewives, mothers and wives, as well as providing food. However, in recent years there has been discussion and study of whether these activities are part of the private and reproductive or actually belong to the public and productive. This is how, from the perspective of gender and the feminist economy, these proposals can be analyzed and the roles that men and women are appointed in a society, in order to determine the forms of behavior in the social, legal, economic, cultural or daily life and trace the origin of such conditions.
\end{abstract}


This research uses the methodology of bibliographic analysis, which collects the concept and theory of the Social and Solidarity Economy and its connection with the Feminist Economy, which, in conclusion, succeed in complementing and committed to a reorganization of work in which the work of women in particular is valued recognizing the construction of a new economy.

Keywords: Feminist Economics, Economics, Gender, Women, Labor.

\section{INTRODUCCIÓN}

En países latinoamericanos como México, Ecuador, Bolivia y Argentina se han venido elaborando iniciativas a través del sector gubernamental, intentando generar desarrollo a través de la aplicación de programas y políticas económicas y sociales, con el único fin de crear empresas para los grupos más desprotegidos los cuales son: las mujeres, los niños, los adultos mayores y los grupos indígenas; sin embargo, estas acciones no han sido suficientes para disminuir la pobreza que prevalece en el país, y principalmente entre la población de mujeres indígenas.

La lucha de los pueblos indígenas al igual que la lucha de las mujeres ha dado resultado, ya que el gobierno se ha visto presionado para crear o modificar marcos jurídicos, instituciones y políticas sociales que se encarguen de salvaguardar los derechos, costumbres y tradiciones de estos grupos, a su vez, desde los grupos sociales desprotegidos han encontrado que para modificar o romper las reglas estructurales y hegemónicas del mercado capitalista que ha ocasionado la división del trabajo por sexos, existe el concepto de Economía Social y Solidaria que propone una forma alternativa de organización al trabajo capitalista y genera redes de solidaridad que permean a cada una de sus integrantes, integra una relación de cuidado al medio ambiente, incluye a mujeres y hombres en los diferentes trabajos y sobre todo, genera cooperación y conexión entre los grupos indígenas involucrados para satisfacer algunas de las necesidades que no logra cubrir el Estado o los gobiernos.

La dinámica del desarrollo local es un proceso en espiral, en el cual de manera constante deben de incorporarse estrategias emergentes a fin de lograr el objetivo planeado. Complementando al desarrollo local, se encuentra un concepto nuevo llamado Economía Solidaria que conlleva a relaciones de reciprocidad e igualdad en el trabajo, además es una forma alternativa al mercado tradicional capitalista.

En países latinoamericanos como los mencionados con anterioridad se ha apostado por proyectos productivos indígenas, los cuales en su mayoría han sido enfocados a mujeres indígenas basándose en sus tradiciones, productos primarios que se producen en sus localidades, habilidades y experiencia. Estos grupos de mujeres han encontrado una nueva forma de organizar el trabajo, la distribución y el consumo de diversos productos hechos por manos indígenas, además, encontrar la revalorización del trabajo conocido como reproductivo. 
El que los proyectos productivos al ser evaluados registren una mayor participación de las mujeres en este ámbito, significa que las mujeres no solo evalúan su participación con el único fin de obtener recursos económicos, sino que también las mueve el aprendizaje, la convivencia, el ser independientes, sentirse más fuertes, y valorar su capacidad de conocimiento para realizar actividades de diversa índole. En combinación, la Economía Social y Solidaria con la Economía Feminista abre un panorama que permite analizar el sentido de producción/reproducción, además de poder observar los cruces interseccionales, por ejemplo, el de ser indígena, pobre y mujer.

\section{METODOLOGÍA}

Derivado del estudio documental y analítico que se desprende de la Economía Social y Solidaria y de la Economía Feminista, en la que se persigue el objetivo de la construcción de una nueva economía que impulse a las mujeres de forma equitativa, en los ámbitos privados y públicos, mediante valores de reciprocidad. En ese sentido, el método que se emplea en esta investigación es el método cualitativo y como herramienta la recolección de datos bibliográficos.

\section{RESULTADOS}

\subsection{DEFINIENDO EL MODELO DE ECONOMÍA SOCIAL SOLIDARIA}

En primer lugar, se retoma el porqué de utilizar el concepto "modelo" para referirse a este tipo de economía, la razón, es porque este término es entendido como "una representación abstracta, conceptual o grafica de fenómenos, sistemas o procesos. Un modelo permite visualizar los elementos generales y las relaciones existentes entre ellos. (Lara y Maldonado, 2014, p. 12) De esta forma permite analizar el sistema económico neoliberal.

Ahora, definido el porqué de utilizar el término modelo, la investigación se enfocará en explicar qué es la Economía Social y Solidaria; este modelo de desarrollo emerge durante el siglo XIX como una respuesta para las condiciones económicas y las necesidades insatisfechas que estaban a cargo del Estado, por lo tanto, surge la necesidad de crear relaciones de solidaridad en las actividades económicas. El devenir de la Economía Social lo podemos encontrar en Europa, específicamente en Francia en el año de 1830 cuando el economista liberal Charles Dunoyer en su obra el Traité d'économie sociale en el cual hacía referencia a una economía con orientación moral. Y es entonces, en Europa donde se acuñe principalmente el término Economía Social y donde se ha trabajado ampliamente en la reestructuración y fortalecimiento de las Instituciones que la conforman. El Centre International de Recherches et D'information sur L'économie Publique, Sociale et Coopérative (CIRIEC), definen a la Economía Social como: 
Conjunto de empresas privadas organizadas formalmente, con autonomía de decisión y libertad de adhesión, creadas para satisfacer las necesidades de sus socios a través del mercado, produciendo bienes y servicios, asegurando o financiando y en las que la eventual distribución entre los socios de beneficios o excedentes así como la toma de decisiones, no están ligados directamente con el capital o cotizaciones apostados por cada socio, correspondiendo un voto a cada uno de ellos. La Economía Social también agrupa a aquellas entidades privadas organizadas formalmente con autonomía de decisión y libertad de adhesión que producen servicios de no mercado a favor de las familias, cuyos excedentes, si los hubiera, no pueden ser apropiados por los agentes económicos que las crean, controlan o financian (Monzón, 2006, p. 15)

En esta definición se pueden encontrar dos tipos de empresas privadas: 1) la empresa de Economía Social y 2) la empresa de capitales; la primera la toma de decisiones no se ve afectada por el capital, sino del voto individual y autónomo de cada persona que está asociada de forma libre, y en la segunda, el derecho al voto se adquiere a partir de la participación en el capital de la empresa.

Seguidamente, la Economía Solidaria nace según algunos autores como Levesque y Mendell, (2003), en Francia y en América Latina a partir de 1960 como una propuesta de la sociedad civil y de movimientos sociales, y que posteriormente incluiría la participación del Estado. De esta manera, en Europa se concibe a la Economía Solidaria como compatible con el Estado y el mercado, mientras que en América Latina se piensa como una propuesta de transformación social como alternativa al neoliberalismo y representa una respuesta a la crisis social. A la Economía Solidaria se le puede definir como:

Formas de organización económica que adoptan los valores de la democracia llevados hasta las últimas consecuencias dentro del área económica: de que todos somos iguales, todos tenemos el mismo poder de decisión, si es necesario delegar todos votan por igual y las personas que los representan tienen que someterse a la voluntad general, caso contrario, son destituidas para que otras sean elegidas. Entonces, Economía Solidaria es una forma democrática e igualitaria de organización de diferentes actividades económicas (Singer, s.f., p. 4)

Cabe resaltar que en la definición de los dos tipos de economía destacan los valores de democracia e igualdad en la toma de decisiones. Finalmente agrupando ambas, el término Economía Social y Solidaria es nuevo, pues es recientemente utilizado en el último cuarto del siglo XX, y se ha definido de varias formas dependiendo de los lugares en los que se ha desarrollado, por ejemplo, en Argentina se pueden abordar los trabajos de Abramovich y Vázquez (2006), en los cuales se expone que la Economía Social y Solidaria es una propuesta política con un enfoque teórico - practico, que rechaza la economía capitalista, que genera equidad, y a través de esta propuesta se busca superar la exclusión social ${ }^{1}$, pero, hay coincidencias al rechazar la economía capitalista, la cual ha generado prácticas antidemocráticas y

\footnotetext{
${ }^{1}$ Hay más propuestas como la de Coraggio (2011), donde aborda cuestiones que incluyen en un régimen del Buen Vivir, mismo que se vincula con la solidaridad, y al contrario de otras propuestas, esta no se presenta como una propuesta de desarrollo sino como una alternativa al desarrollo neoliberal, y que el término Economía Social es innecesario porque de hecho la economía es social.
} 
agravado la pobreza y la desigualdad, sino que más bien es una propuesta alternativa al desarrollo neoliberal.

Además, esta propuesta nueva se concibe mejor en países sudamericanos donde "hace menos de dos décadas atrás se empieza a reconocer "oficialmente" su diversidad étnico-cultural; una diversidad histórica enraizada en políticas de exterminio, esclavización, deshumanización, interiorización y también en la supuesta superación de lo indígena y negro" (Batalla, 1991, p. 62), asimismo, como proyecto alterno al capitalismo y como ejemplo está el caso de Bolivia como precursor en América Latina, en donde la diversidad de culturas predomina y ha modificado la forma de vida, las instituciones y la ley, por lo tanto, a través de estos cambios se ha podido conectar la Economía Social y Solidaria y la interculturalidad, generando mayor desarrollo sin vulnerar los derechos de las diferentes culturas que conviven en el territorio. Cabe resaltar que, "la economía solidaria permanece en comunidades tradicionales (indígenas, quilombolas y campesinas) cuando éstas organizan el trabajo y el manejo del territorio con respeto a todas y todos y a la naturaleza" (Nobre, 2015, p. 18). A continuación, se presenta el cuadro 1 conformado por algunas definiciones de la Economía Social Solidaria con el fin de aclarar las dudas. 
Tabla 1. Definiendo a la Economía Social Solidaria

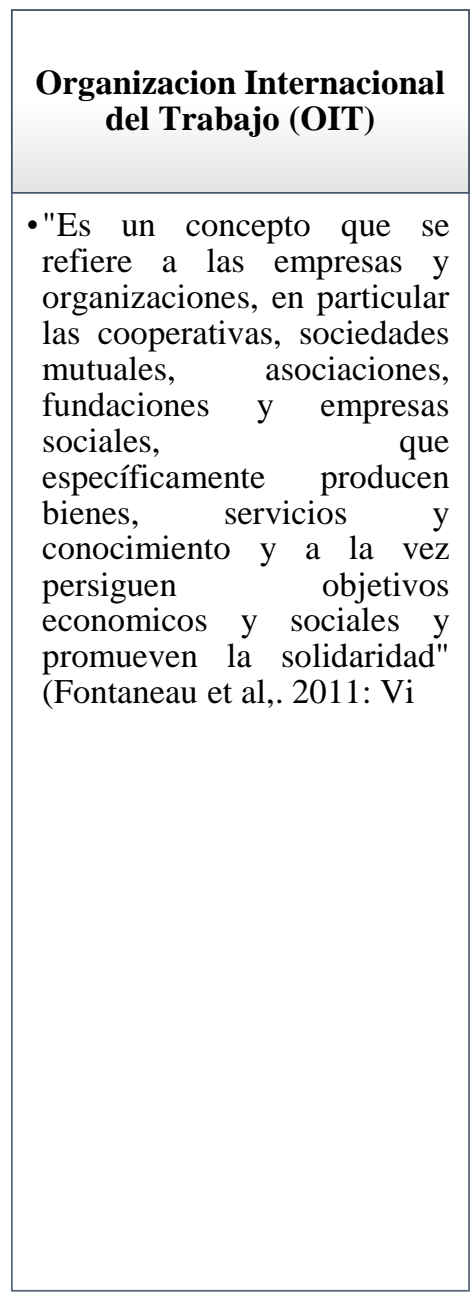

\begin{tabular}{|c|}
\hline $\begin{array}{c}\text { Espacio Nacional de la } \\
\text { Economia Social y Solidaria } \\
\text { (ENESS) }\end{array}$ \\
\hline 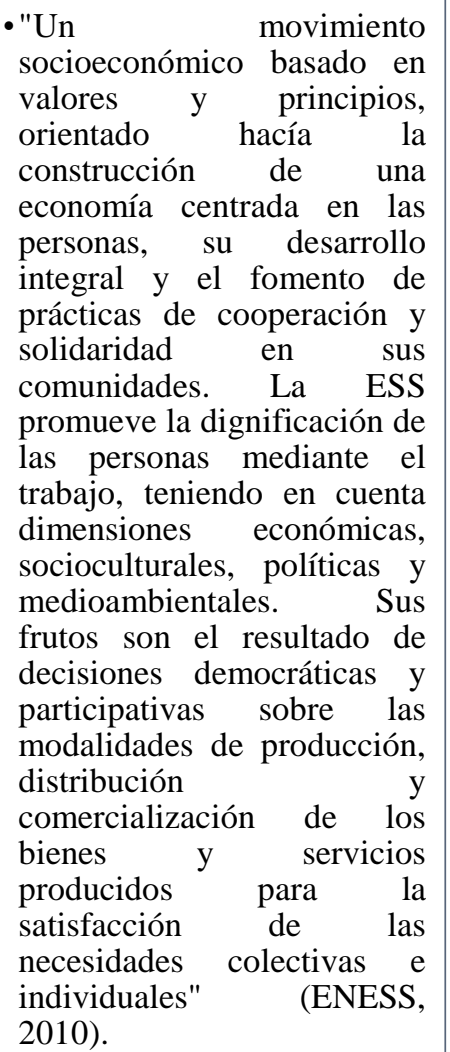 \\
\hline
\end{tabular}

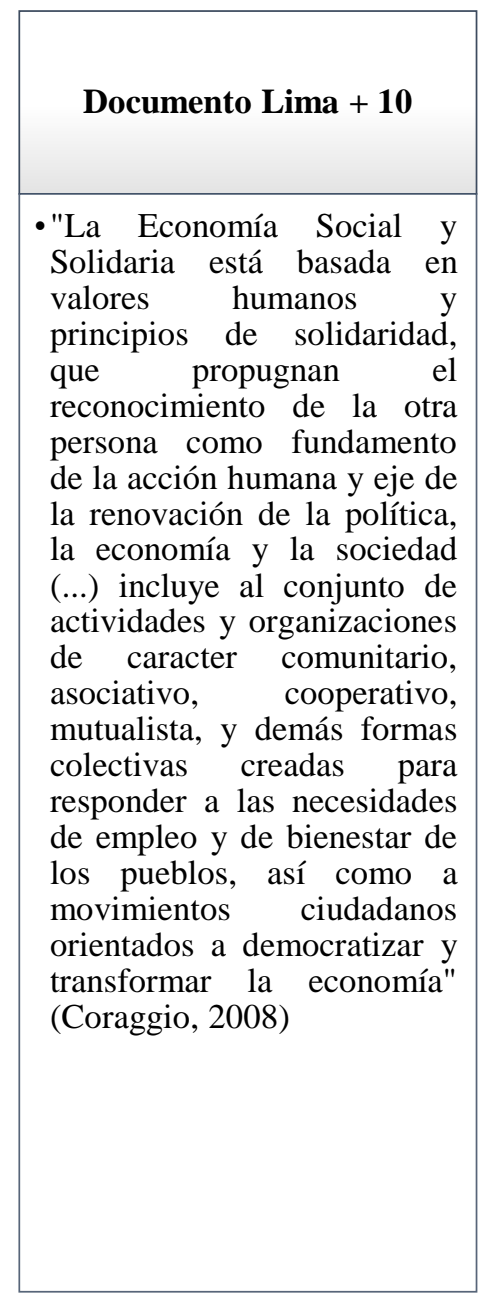

Nota: Lara y Maldonado, (2014) con base en Fonteneau et al., 2011:VI; ENESS, 2010; Coraggio, 2008.

Al haber conceptualizado y dado las características de la Economía Social y Solidaria, también es importante señalar algunas de las formas organizacionales que la acompañan, en este sentido Fonteneau (2011), señala que la Economía Social y Solidaria comprende formas específicas de empresas y organizaciones. Las cooperativas, sociedades mutuales, asociaciones y empresas sociales son los tipos más comunes, pero no son los únicos al ser grupos de interlocutores dinámicos y en evolución en los cuales todos promueven y administran organizaciones económicas centradas en las personas.

Para este tipo de empresas y organizaciones, queda en segundo plano, pero no menos importante el aspecto económico y su rentabilidad, por eso, en muchas ocasiones se las caracteriza como la economía de los pobres y por esta razón, es que es importante integrar a diferentes personas pertenecientes a diversos estratos sociales, lo que causara una efectiva viabilidad económica y creara la base para la solidaridad.

Sin embargo, algunos afirman que la teoría de la Economía Social y Solidaria tiende a ser utopista al poner en duda la existencia de verdaderas relaciones de solidaridad y reciprocidad en las comunidades o sectores populares. Por lo cual, autores como Martínez (2009) mencionan que debe de haber una 
heterogeneidad en las formas productivas para funcionar de una forma mejor que las mismas empresas capitalistas. Además, como lo dice Caillé (citado en Martínez, 2009, p. 112) resaltan dos posiciones de la Economía Social y Solidaria: entre aquellos para los cuales la Economía Solidaria busca reemplazar integralmente el capitalismo y aquellos para quienes representa una forma de economía mixta.

Con todo, la Economía Social y Solidaria, genera ingresos y desenvuelve las capacidades de auto organización y cooperación para solucionar problemas y necesidades tanto personales como comunales o de la misma sociedad civil, también, promulgan valores de solidaridad y moral económica en el consumo y producción de manera responsable. En la actualidad, existen diversos tipos de organizaciones que fomentan la Economía Social y Solidaria y con las características antes descritas que las hacen diferentes a las organizaciones públicas o privadas dado su enfoque solidario.

El sector social de la economía se ha abandonado, por lo cual, se ha priorizado el sector público y el privado. Esta propuesta, se cataloga como necesaria para una transformación que contribuya al mejoramiento de la calidad de vida de las personas. Asimismo, es un proyecto que poco a poco gana espacio entre las vertientes importantes de la economía y de gran impacto en la política y lo social. En primer lugar, permite realizar políticas públicas que realmente favorezcan a los beneficiarios y social porque la concurrencia de diversas organizaciones orientadas hacia la Economía Social y Solidaria ha aportado conocimientos y campo de estudio, que también, genera valores y prácticas basadas en la solidaridad. Igualmente, demuestra su eficiencia y eficacia al mostrar casos de éxito en varias partes del mundo.

En resumen, se afirma que la Economía Social y Solidaria es una propuesta alterna al mercado capitalista que recupera los aspectos sobre el cuidado de las comunidades tradicionales e incrementa la participación de las y los miembros que a su vez generan redes de cooperación, sobre todo cuando el Estado o los gobiernos se encuentran en crisis, rompiendo con los roles preestablecidos.

Entonces, dicha propuesta difunde que se

aspire a la construcción de relaciones de producción, de intercambio y de cooperación que motiven la suficiencia, más que la eficiencia y la calidad, sustentadas en la solidaridad; esto es, dejar de lado la tendencia dominante de las empresas a la maximización de las utilidades a costa de largas jornadas laborales cuya remuneración se precariza de manera constante (Lara y Maldonado, 2014, p. 25)

Un avance que se considera necesario es la creación de políticas públicas focalizadas a grupos particulares que les permita articular redes de apoyo para minimizar las consecuencias del mercado capitalista que ha agudizado los problemas sociales en lugar de solucionarlos. Los grupos pertenecientes a la Economía Social y Solidaria necesitan fortalecerse e involucrarse para ampliar las posibilidades que dan las políticas públicas más allá de lo ya institucionalizado, seguir reconstruyendo formas de 
valorización del trabajo y no parar la búsqueda de la autonomía personal y colectiva encontrando nuevas formas de organización, haciendo también participes a hombres, mujeres y gobierno para convertirse en sujetos políticos activos y participativos.

\section{DISCUSIÓN}

\subsection{EL GÉNERO EN EL MODELO DE LA ECONOMÍA SOCIAL Y SOLIDARIA}

Hablar de género en la actualidad es hablar de mujeres primordialmente debido a su innumerable uso en la política, al emplearlo "como sinónimo de mujeres o de desigualdad social, sin que por lo general se haga precisión conceptual alguna" (Palomar, 2016, p. 43), más bien, retomando el término en un sentido más académico, el género nos permite analizar los papeles que se designan a los hombres y a las mujeres en una sociedad y de esta forma observar las formas de comportamiento en lo social, jurídico, económico, cultural o en la vida cotidiana, además del uso al que se refiere a la construcción cultural de la diferencia sexual. Pero, no se debe de olvidar que "el uso de esta perspectiva permite analizar una de las tantas formas simbólicas de que se vale la cultura para institucionalizar la diferencia entre hombres y mujeres y para poner en escena sus confrontaciones" (Lamas, 1995, p. 336)

Es por ello por lo que, se pueden analizar las maneras en que las instituciones reproducen lo que es femenino o masculino. Género abarca una amplia gama de construcciones sociales, ya que han sido establecidas por una sociedad patriarcal dentro de un proceso histórico, además, ha sido una interpretación desde lo natural y de los rasgos biológicos que identifican a las mujeres por ser aquellas que reproducen la vida y se les han dado espacios determinados de trabajo, por ejemplo, al cuidado de otros. "Una mujer es una mujer. Solo se convierte en doméstica, esposa, mercancía, conejito Playboy, prostituta o dictáfono en determinadas relaciones" (Rubin, 1975, p. 36)

En este contexto, se debe traer a discusión el cómo es que se han designado determinados roles a las mujeres a través del trabajo el cual se encuentra en la lógica capitalista en la producción y la reproducción. El ya tradicional modelo de economía capitalista que ha moldeado la división sexual del trabajo es el principal opresor de las mujeres, ya que esta división ha logrado que los individuos diferenciemos entre funciones que tienen más valor de uso, con esto quiero decir que tiene valor de cambio, lo cual generará plusvalía y acumulación del capital a cambio de fuerza de trabajo.

A diferencia de otras en el caso de las funciones productivas, las funciones reproductivas consisten en aquellas que reproducen las fuerzas de trabajo, estas se quedan restringidas al ámbito privado, en lo familiar, donde "la comida debe ser cocida, las ropas lavadas, las camas tendidas, la leña cortada, etcétera. Por consiguiente, el trabajo doméstico es un elemento clave en el proceso de reproducción del trabajador del que se extrae plusvalía" (Rubin, 1975, p. 40) 
Se ha sostenido que las mujeres son una reserva de fuerza de trabajo para el capitalismo, que los salarios generalmente más bajos de las mujeres proporcionan plusvalía extra al patrón capitalista, que las mujeres sirven a los fines del consumismo capitalista en sus papeles de administradoras del consumo familiar, etcétera (Rubin, 1975, p. 38)

En este sentido, se afirma que cuando una mujer prepara un plato de comida al igual que si trabajara en el campo sembrando algo y no recibe un salario por dicho trabajo, está realizando una actividad económica que contribuyen a la reproducción de la vida y agrega capital a la plusvalía final, este es el origen de la opresión a las mujeres, agregando que el trabajo reproductivo ha sido desvalorizado e invisibilizado y enraizado en la tradición histórica y social, como lo menciona Rubin (1975):

\footnotetext{
Es precisamente ese "elemento histórico y social” lo que determina que una "esposa" es una de las necesidades del trabajador, que el trabajo doméstico lo hacen las mujeres y no los hombres, y que el capitalismo es heredero de una larga tradición en que las mujeres no heredan, en que las mujeres no dirigen y en que las mujeres no hablan con el dios. Es este "elemento histórico y moral" el que proporciono al capitalismo una herencia cultural de formas de masculinidad y femineidad. Es dentro de ese "elemento histórico y moral" que esta subsumido todo el campo del sexo, la sexualidad y la opresión sexual. (p. 42)
}

Por lo tanto, cambiar la economía es necesario y es un trabajo arduo, sobre todo cuando se la pretende guiar hacia valores de solidaridad, reciprocidad, justicia e igualdad. Pero, para ello se tiene de herramienta a la Economía Feminista que va de la mano con la economía social solidaria. "La economía feminista nos impulsa a ampliar lo que se comprende como trabajo, incluyendo no solo lo que es remunerado, sino todo lo que es necesario para la producción de la vida, de los bienes y servicios que necesitamos, de las relaciones, los afectos y los cuidados que todas las personas necesitan a lo largo de sus vidas" (Nobre, 2015, p. 8). Este enfoque permite visualizar los procesos que realmente sostienen a la sociedad que va más allá de la reproducción de la vida necesaria para el trabajo, es más bien una forma nueva de organización en la que se le dé el cuidado a la vida.

Con esta perspectiva, la Economía Social y Solidaria permitirá a través de las relaciones de solidaridad y reciprocidad una reorganización del sistema de producción, en donde el trabajo, el conocimiento y el emprendimiento de las mujeres sea valorizado y que esta nueva forma de organización introduzca al hombre hacia las labores del hogar. La Economía Social y Solidaria, al incluir a las mujeres no solo les da la posibilidad de tener ingresos económicos para logar su independencia, sino que también les da la posibilidad de ser sujetos políticos y ser tomadoras de decisiones lo que implica ser autónomas. Estas redes generadas entre mujeres también contribuyen a la cohesión social y al crecimiento individual y colectivo, ya que la organización colectiva reproductiva provee aprendizaje, autonomía y sociabilidad como ya lo hemos mencionado antes. 
La Economía Social y Solidaria busca superar al capitalismo, y dividir entre lo político y lo económico, ya que en el capitalismo se encuentra que el sistema económico actúa de forma "natural" a través de la ley de oferta y demanda por ejemplo y que de esta forma la toma de decisiones se mantiene entre las elites de poder. Es entonces, que las mujeres pertenecientes a la Economía Social y Solidaria "se constituyen en un espacio de intermediación entre Estado, mercado y familia" (Nobre, 2015, p. 17). Estas mujeres buscan dar solución y crear nuevas relaciones entre hombres y mujeres, aplican la justicia a través de la negociación, de los derechos y de la comunidad.

También, la participación de las mujeres promueve su participación a movimientos sociales de los grupos productivos que se manifiestan y exigen a los gobiernos subsidios, mas formas de participación entre otras, y quizás una de las más importantes es que se hacen notar.

Para finalizar, las prácticas de la Economía Social y Solidaria proponen una nueva forma de organización cuando el Estado y los gobiernos no logran satisfacer las necesidades de los ciudadanos, es especial de los indígenas o de los grupos vulnerables, esta crisis o ruptura de la economía capitalista ha dado cabida a que estos grupos se organicen a través de sociedades cooperativas, empresas sociales comunitarias, radios comunitarias entre otras, en donde se las mujeres se han apoderado de dichos espacios. Esto solo es un paso hacia la economía política de la resistencia en donde las mujeres aportan sus experiencias a la producción colectiva al satisfacer las necesidades cotidianas.

\section{CONCLUSIONES}

La Economía Social y Solidaria como alterna a la economía capitalista recupera los aspectos sobre el cuidado de las comunidades tradicionales e incrementa la participación de las mujeres en movimientos sociales que a su vez generan redes de cooperación, sobre todo cuando el estado o los gobiernos se encuentran en crisis, rompiendo con los roles preestablecidos y quebrantando el orden establecido entre lo productivo y lo reproductivo.

Un avance que se considera necesario, en particular en el caso de los países latinoamericanos, es la creación de políticas públicas focalizadas a grupos particulares que les permita articular redes de apoyo para minimizar las consecuencias del mercado capitalista que ha agudizado los problemas sociales en lugar de solucionarlos. Los grupos de mujeres en la Economía Social y Solidaria necesitan fortalecerse e involucrarse para ampliar las posibilidades que dan las políticas públicas más allá de lo ya institucionalizado, seguir reconstruyendo formas de valorización del trabajo y no parar la búsqueda de la autonomía personal y colectiva encontrando nuevas formas de organización, haciendo también participes a hombres y mujeres para convertirse en sujetos políticos activos y participativos. 


\section{REFERENCIAS BIBLIOGRÁFICAS}

Abramovich, A., Vázquez G. (2006), "Experiencias de Economía Social y Solidaria en la Argentina", Karina Forcino y Victoria Basualdo (coords.), en Transformaciones en la economía argentina: pasado reciente y perspectivas, Instituto del Desarrollo Humano-UNGS, Argentina.

Batalla, G. (1991). Pensar nuestra cultura. En Diálogos en la acción, primera etapa, 2004, 117-134. Recuperado de http://www.ilam.org/ILAMDOC/sobi/Guillermo\%20Bonfil_PensarnuestraculturaPat.pdf

Coraggio, J. (2011). "Economía social y solidaria. El trabajo antes que el capital”. Ecuador: Ediciones Abya-Yala.

Coraggio, L. (2008), La Economía Social y Solidaria como estrategia de desarrollo en el contexto de la integración regional latinoamericana. en http://www.econo.unlp.edu.ar/uploads/docs/economia_social_solidaria_america_latina_jose_luis_corag gio.\%20Am\%C3\%A9rica\%20Latina\%20-\%20Coraggio.pdf.

Fonteau, B. (2011). Economía Social Solidaria: nuestro camino común hacia el trabajo decente. Documento de trabajo 2010. Centro Internacional de Formación de la Organización Internacional del Trabajo. Recuperado de: https://www.ilo.org/wcmsp5/groups/public/---ed_emp/---emp_ent/--coop/documents/instructionalmaterial/wcms_166369.pdf

Gómez, G., Candelaria, M. (2014) Modelos emergentes de desarrollo en la economía social y solidaria. Querétaro, México: MAPorrúa

Lamas, M. (1995). Usos, dificultades y posibilidades de la categoría de "género". En El género: la construcción cultural de la diferencia sexual (327-366). México: PUEG-UNAM/Porrúa, 1996

Lara, G. y Maldonado, M. (2014). Modelos emergentes de desarrollo en la economía social y solidaria. Editorial: Porrúa.

Martínez, L. (2009) La Economía Social y Solidaria ¿Mito o realidad? Íconos. Revista de Ciencias Sociales, núm. 34. p. 107-113

Monzón, J. (2006). Economía Social y conceptos afines: fronteras borrosas y ambigüedades conceptuales del Tercer Sector. CIRIEC-España, Revista de Economía Pública, Social y Cooperativa. Num. 56. 9-24. http://www.redalyc.org/articulo.oa?id=17405602

Nobre, M., Faria, N. y Moreno, R. (2015) Las mujeres en la construcción de la economía solidaria y la agroecología. Textos para la acción feminista. San Paulo, Brasil: Sempreviva Organizaçåo Feminista Palomar, C. (2016). Veinte años de pensar el género. Debate feminista, 52, 34-49.

Rubin, G. (1975). El tráfico de mujeres: Notas sobre la 'economía política' del sexo. En Marta Lamas (Comp.), El género: la construcción cultural de la diferencia sexual (35-97). México: PUEGUNAM/Porrúa, 1996.

Singer, P. (s.f.), Economía Solidaria: posibilidades y desafíos. Recuperado en htpp://www.dhl.hegoa.ehu.es/ficheros/0000/0179/economia_solidaria_posibilidades_y_desafios.pdf 